\title{
Magnetization dynamics with a spin-transfer torque
}

\author{
Z. Li and S. Zhang \\ Department of Physics and Astronomy, \\ University of Missouri-Columbia, Columbia, MO 65211
}

(Dated: November 1, 2018)

\begin{abstract}
The magnetization reversal and dynamics of a spin valve pillar, whose lateral size is $64 \times 64$ $\mathrm{nm}^{2}$, are studied by using micromagnetic simulation in the presence of spin transfer torque. Spin torques display both characteristics of magnetic damping (or anti-damping) and of an effective magnetic field. For a steady-state current, both M-I and M-H hysteresis loops show unique features, including multiple jumps, unusual plateaus and precessional states. These states originate from the competition between the energy dissipation due to Gilbert damping and the energy accumulation due to the spin torque supplied by the spin current. The magnetic energy oscillates as a function of time even for a steady-state current. For a pulsed current, the minimum width and amplitude of the spin torque for achieving current-driven magnetization reversal are quantitatively determined. The spin torque also shows very interesting thermal activation that is fundamentally different from an ordinary damping effect.
\end{abstract}

PACS numbers: 


\section{INTRODUCTION}

Recently, there is considerable interest in the phenomenon of spin-polarized current induced magnetization switching. This phenomenon was first suggested by Berger [1] and Slonczewski 2], based on a rather general argument: for a system consisting of itinerant electrons and local moments, the total angular momentum is conserved even for the system out of equilibrium, and thus the divergence of the spin current of itinerant electrons must be accompanied by an equal and opposite change of the angular momentum of the local moment. This change is equivalent to a spin torque acting on the local moment. Since then, there are many theoretical [3, 4, 5, 6, 7, 8] and experimental efforts [9, 10, 11, 12, 13, 14] to understand the microscopic origins. In this paper, we do not discuss the microscopic origins and the detailed formalism of the current-induced spin torque. Instead, we consider the consequences of the spin torque on the dynamics of the local moment of a spin valve structure.

Regardless of the detailed physics involved microscopically, the effect of the spin angular momentum transfer can be captured by an additional term in the macroscopic LandauLifshitz-Gilbert (LLG) equation,

$$
\frac{d \mathbf{M}}{d t}=-\gamma \mathbf{M} \times \mathbf{H}_{e f f}+\frac{\gamma a_{J}}{M_{s}} \mathbf{M} \times\left(\mathbf{M} \times \hat{\mathbf{M}}_{p}\right)+\frac{\alpha}{M_{s}} \mathbf{M} \times \frac{d \mathbf{M}}{d t}
$$

where $\gamma$ is the gyromagnetic ratio, $\mathbf{M}$ is the magnetization vector of the free layer, $\hat{\mathbf{M}}_{p}$ is the unit vector whose direction is along the magnetization of the pinned layer, $M_{s}$ is the saturation magnetization, $\mathbf{H}_{e f f}$ is the effective magnetic field including the external field, the anisotropy field, the exchange field, the demagnetization field and the random thermal field whose form will be discussed in the next section. The term proportional to $a_{J}$ is the novel spin torque term, and the last term is the Gilbert damping term. It is noted that $a_{J}$ has dimensions of magnetic field.

Experimental verification of the spin torque has been carried out in magnetic nanowires 15], spin valve pillar structures [10, 12, 13], point-contact geometry [9, 16], and magnetic tunnel junctions [11, 17]. The convincing observation of these experiments is that there exists a critical current density above which the magnetization can be switched back and forth. Other properties, such as thermal effects [18], also agree with the spin torque term in Eq. (1). Theoretical analysis has been mostly confined to a single domain structure 
[19, 20]. Both analytical and numerical solutions indicate that the magnetization reversal becomes very complicated even for a single domain structure. For example, the hysteresis for a fixed current density may display a precessional mode, and there is a possibility of an inverse hysteresis (the magnetization along the direction of applied field decreases when one increases the applied field). Miltat et al. [21] made a step forward in relaxing the single domain assumption by studying the magnetization reversal for an "S" state and a "leaf" state [22]; these are typical equilibrium domain structures of a submicron thin film. They concluded that the effect of the current is quite different for these two structures even though they have nearly the same magnetic energy.

The spin torque is fundamental new from the pure precessional term (first term in Eq. (1)) and from the damping term (last term in Eq. (1)). The precession term conserves the magnetic energy and it determines the precessional frequency of the magnetization dynamics. The damping term makes the magnetic system relax to a local energy minimum, i.e., it dissipates the energy during magnetization dynamics. The spin torque term, however, can have both effects: it can be a source of precessional motion as an effective field and it can serve as damping (or anti-damping) sources. It is this dual function that motivates us to study magnetization dynamics in a realistic magnetic nanostructure. In this paper, we perform an extensive study on the effect of the spin torque from the LLG equation, Eq. (1). The paper is organized as follows. In Sec. II, the model geometry of a spin valve pillar is defined and the method for micromagnetic simulation is outlined. Next, we calculate hysteretic and dynamical properties in the presence of the spin torque; in particular, we analyze precessional states in detail. We also present the results for a nonsteady-state current in Sec. III. Finally we summarize what are the most interesting features of magnetization dynamics in the presence of the current-induced spin torque.

\section{MICROMAGNETIC MODEL WITH THE SPIN TORQUE}

To mimic the experimental geometry performed by the group at Cornell [12], we define our structure in Fig.1. The electrical current flows perpendicular to the plane of the layer. The coordinate axes are so chosen that the $x$ and $y$ axes are in the plane of the layer and the $z$-axis is perpendicular to it. A spin-polarized current $j$ enters the spin valve pillar in the $z$ direction and we assign the positive value of the parameter $a_{J}$ in Eq. (1) for the 
current flowing from the thicker ferromagnetic layer to the thinner one. The current induced magnetic field is ignored.

Since we focus our study on magnetization dynamics of the free layer, we assume that the pinned ferromagnetic layer is held fixed at the direction of the easy axis of the free layer, i.e., in positive $x$ direction. The following materials parameters for the free layer are used: the lateral size is $64 \mathrm{~nm} \times 64 \mathrm{~nm}$, the thickness is $2.5 \mathrm{~nm}$, the uniaxial anisotropy field $H_{K}$ is $500(\mathrm{Oe})$, and the saturation magnetization $4 \pi M_{s}=12,000$ (Oe). These parameters are reasonably consistent with the experiments by the Cornell group [12]. We note that the choice of the small layer thickness has two advantages: the magnetization direction in the thickness direction will be uniform, i.e., a 2-d micromagnetics modeling is sufficiently accurate and the spin torque per unit volume is large for a fixed current density (since the total spin torque will be almost independent of the free layer thickness within the applicability of several theoretical models).

With above specified parameters, we begin our simulation by laterally dividing the free layer into an $\mathrm{N} \times \mathrm{N}$ grid. In most cases presented in the paper, we choose $\mathrm{N}=16$ so that the grid spacing is $D=4.0 \mathrm{~nm}$. Our goal is to calculate magnetization dynamics as the external magnetic field, the spin torque, and the damping parameter vary. The magnitude of the spin torque is proportional to the current density. Here we have chosen the unit of $a_{J}$ in Oesteds. The numerical value of $a_{J}$ has been estimated [2, 7]: for the current density $j=10^{8} \mathrm{~A} / \mathrm{cm}^{2}, a_{J}$ in Eq. (1) is about $1 \mathrm{kOe}$. This conversion between $a_{J}$ and the current density is, however, irrelevant for us since we have written our results in terms of $a_{J}$ and thus we do not specify absolute values of the current density. The Gilbert damping constant $\alpha$ in Eq.(1) is not accurately known in a spin valve system. Among many physical sources for the damping, there is an additional contribution at the interface. Recent studies [23, 24, 25] for ultrathin films show that the damping constant $\alpha$ is much enhanced when a nonmagnetic metal is deposited on the ferromagnetic film. For $C u / C o$ bilayers, the damping constant is considerably larger than that of bulk value of $C o$. With this difficulty in choosing $\alpha$, we will vary it from 0.001 to 0.2 to address the damping dependence of the magnetization dynamics.

The effect of temperature on the dynamical behavior is also included in our simulation by adding a random thermal field to the effective magnetic field. The thermal field $H_{t h, i}^{\xi}(t)$ at each site $i$ is assumed to be an independent Gaussian random function with its zero mean and no correlation, i.e., $<H_{t h, i}^{\xi}(t) H_{t h, j}^{\eta}\left(t^{\prime}\right)>=2 G \delta_{i j} \delta_{\xi \eta} \delta\left(t-t^{\prime}\right)$, where $i, j$ are the cell 
indexes, $\xi, \eta$ represents three Cartesian components, $G=\alpha k_{B} T / \mu_{0} \gamma M_{s} \nu$, and $\nu$ denotes the discretization volume of the computational cells. By adding the thermal activation, the Landau-Lifshitz-Gilbert equation is converted into a stochastic differential equation with multiplicative noise. The integration of the stochastic Landau-Lifshitz-Gilbert equation is performed by employing the stochastic Heun method, by starting from a given initial configuration, and updating recursively the state of the system, $\mathbf{M}(t) \rightarrow \mathbf{M}(t+\delta t)$ [26, 27, 28]. The Heun scheme is a good compromise between numerical stability and computational complexity. In general, the statistical error of Heun scheme is made arbitrarily small by averaging over a sufficiently large number of stochastic trajectories. We do not carry out $\delta t \rightarrow 0$ limiting procedure but we employ a small discretization time interval, i.e., we use $\delta t=0.3 p s$ throughout the paper.

\section{RESULTS}

Many interesting features have already been shown by simply assuming the free layer is a single domain. As studied by Sun 19] and Bazaliy et al. [20], the spin torque term in LLG makes the magnetization dynamics quite complicated. The complication arises from the observation of the LLG equation that the spin torque is fundamentally different from the effective field term and from the damping term. The effective field term, first term in Eq. (1), can be derived from the energy derivative with respect to the local magnetization vector,

i.e., $\mathbf{H}_{e f f}=-\frac{\partial E(\mathbf{M}(\mathbf{r}))}{\partial \mathbf{M}(\mathbf{r})}$ where $E(\mathbf{M}(\mathbf{r}))$ is the magnetic energy including the exchange energy between the neighboring cells, the magnetostatic (dipole) energy, the (uniaxial) anisotropy energy, and the Zeeman energy. The damping term, which can not be written as the energy derivative, selects the magnetization path such that the local magnetization always moves into lower energy states, i.e., the system is looking for an energy minimum. The spin torque neither behaves as an effective field which conserves magnetic energy nor the damping term which dissipates the energy during the motion of magnetization. The spin torque can increase or decrease the magnetic energy. This leads to some interesting solutions with stable precessional states. We will show that these stable precessional states exist for finite temperature and for non-single-domain structures.

Another interesting effect of the spin torque is the appearance of the inverse hysteresis loop for certain range of the magnetic field, i.e., the magnetization along the direction of 
the magnetic decreases as one increases the magnetic field. To see this, let us consider the time-independent solution, $\frac{d \mathbf{M}}{d t}=0$. Equation (1) becomes

$$
\boldsymbol{\Gamma} \equiv \mathbf{M} \times \mathbf{H}_{e f f}-\frac{a_{J}}{M_{s}} \mathbf{M} \times\left(\mathbf{M} \times \hat{\mathbf{M}}_{p}\right)=0 .
$$

For a single domain, we explicitly write $\mathbf{H}_{e f f}=\left(H_{e x t}+H_{K} M_{x}\right) \mathbf{e}_{x}-4 \pi M_{s} M_{z} \mathbf{e}_{z}$ and $\hat{\mathbf{M}}_{p}=\mathbf{e}_{x}$. By placing these expressions into Eq. (2), we find

$$
M_{z}\left[\left(H_{e x t}+H_{K} M_{x}\right)^{2}+4 \pi M_{s} M_{x}\left(H_{e x t}+H_{K} M_{x}\right)+a_{J}^{2} M_{x}^{2}\right]=0
$$

The above equation indicates that $M_{z}=0$ is always a solution, i.e., the magnetization is in the plane of the layer. However, there are other possible solutions with $M_{z} \neq 0$. From Eq. (3), we have

$$
\left(H_{e x t}+H_{K} M_{x}\right)^{2}+4 \pi M_{s} M_{x}\left(H_{e x t}+H_{K} M_{x}\right)+a_{J}^{2} M_{x}^{2}=0 .
$$

Thus two solutions can be immediately identified,

$$
H_{e x t}^{ \pm}=-H_{K} M_{x}-2 \pi M_{s} M_{x} \mp \sqrt{\left(2 \pi M_{s} M_{x}\right)^{2}-a_{J}^{2} M_{x}^{2}} .
$$

For $2 \pi M_{s} \gg a_{J}$, two solutions are further simplified as $H_{e x t}^{+}=-M_{x}\left(H_{K}+4 \pi M_{s}-\frac{a_{J}^{2}}{4 \pi M_{s}}\right)$ and $H_{e x t}^{-}=-M_{x}\left(H_{K}+\frac{a_{J}^{2}}{4 \pi M_{s}}\right)$. These solutions show that the magnetic field and the magnetization $M_{x}$ have opposite sign, i.e., an inverse hysteresis. However, we need to point out that these solutions do not always stable compared to the simple solution $M_{z}=0$. When one examines the stability condition, it is found that the above solutions $H_{\text {ext }}^{ \pm}$are possible for a finite range of $a_{J}[20]$.

The third interest feature is that there is a critical current density, or a critical value of $a_{J}$, above which the spin torque can be used to switch one magnetic configuration to another, for example, to switch the magnetization from parallel to antiparallel alignment of the two magnetic layers. If one assumes the single domain of the free layer, the critical value can be readily deduced from the average energy variation rate [2, 19],

$$
\left(a_{J}\right)_{c r i t}= \pm \alpha\left(2 \pi M_{s}+H_{K}\right)+\alpha H_{e x t}
$$

This simple relation indicates that the current-magnetization hysteresis loop displays a jump when the current is swept through the critical current. One might raise a question that why the critical current depends on demagnetization factor $4 \pi M_{s}$ in Eq. (6). The answer is 
that the magnetization reversal involves a significant out-of-plane component of the magnetization. Once the magnetization rotates out of the plane during a reversal process, the demagnetization factor is added to the effective field in LLG equation. Thus, the damping term $\left[\alpha \mathbf{M} \times\left(\mathbf{M} \times \mathbf{H}_{e f f}\right)\right]$, which is also proportional to the effective field, contains the demagnetization factor. To overcome the damping, the critical spin torque is, therefore, proportional to the demagnetization factor.

The above features derived from the single domain structure show unique characteristics of the spin torque. To further explore the spin torque effect, one needs to go beyond the hysteresis analysis, i.e., one should look at the detailed magnetization dynamics, and one must relax the assumption of the single domain. We now present our results in the following sections.

\section{A. Hysteresis loops with spin torques}

Without the spin torque, the hysteresis loop is almost a perfect square with the coercive field $H_{c}=H_{K}$, i.e., the hysteresis loop behaves as a Stoner-Wohlfarth particle with the applied magnetic field parallel to the easy axis. When a spin torque is added on, the hysteresis loops display three distinct features. For a small current density, $\left|a_{J}\right|<2 \pi \alpha M_{s}$, the hysteresis loops are not affected by the spin torque, see Fig. 2a. When the current density increases to intermediate values, the hysteresis loops begin to show some precessional states; those states are shaded in black, indicating that the magnetization is never converged to a final fixed direction; instead, it goes into a stable precession and we will further discuss those precessional states in later sessions. With this intermediate strength of the spin torque, the precessional magnetization is oscillating around the easy axis. Just before the appearance of the precessional states in Fig. (2b), two irreversible jumps $H^{-}$and $H^{+}$occur; they can be again understood from the single domain solution. From the expression below Eq. (5), one can identify

$$
H^{-}=-H_{K}-\frac{a_{J}^{2}}{4 \pi M_{s}}
$$

On the other hand, for the field swept from negative $x$ to positive $x$ direction, one can use Eq. (6) to solve for $H^{+}$

$$
H^{+}=-\frac{\left|a_{J}\right|}{\alpha}+H_{K}+2 \pi M_{s}
$$


We note that $H^{+}$defined here is different from $H_{e x t}^{+}$of Eq. (6) because the latter is for a high magnetic field. In the present case, the irreversible jump at $H^{+}$occurs at much smaller field. From Eqs. (7) and (8), the coercivity $H_{c}=\left(H^{+}-H^{-}\right) / 2=H_{K}-\frac{1}{2}\left(\frac{\left|a_{J}\right|}{\alpha}-2 \pi M_{s}\right)+\frac{a_{J}^{2}}{8 \pi M_{s}}$ decreases with the spin torque and the loops shift $\delta H=\left(H^{+}+H^{-}\right) / 2=-\frac{1}{2}\left(\frac{\left|a_{J}\right|}{\alpha}-2 \pi M_{s}\right)-$ $\frac{a_{J}^{2}}{8 \pi M_{s}}$ appears, i.e., the spin torque supplies a bias field to the loop.

When the current density is further increased such that $a_{J}>\alpha\left(2 \pi M_{s}+2 H_{K}\right)$, the loops become dramatically different from those of low current densities. The loops show multiple jumps and the precessional solutions expand to a large range of the magnetic field (note the scale difference in Fig. 2c). In addition, there is the region in the loop where the magnetization increases with decreasing magnetic field, e.g., when the magnetic field decreases from $-9,900$ (Oe) to $-12,4000$ (Oe), the magnetization $M_{x}$ increases from 0.78 to 0.99. This unusual solution at high field is consistent with the single domain solution given by Eq. (4), i.e., we can solve for $M_{x}$ from Eq. (4),

$$
M_{x}=\frac{-H_{e x t}\left(H_{K}+2 \pi M_{s}-\sqrt{H_{K}^{2}+\left(2 \pi M_{s}\right)^{2}-a_{J}^{2}}\right.}{H_{K}^{2}+4 \pi M_{s} H_{K}+a_{J}^{2}} .
$$

The solutions are stable; we have varied temperatures and confirmed that these states exist for all temperatures.

\section{B. Magnetization-current hysteresis loops at finite external fields}

In Fig. 3, we show M-I hysteresis loops (magnetization as a function of the spin torque) for several different external fields. When the external magnetic field is smaller than the anisotropy field, i.e., $H_{e x t}<H_{K}$, the M-I loop is almost square in shape and the critical spin torque is very close to the analytical expression given by Eq. (6). For example, the loop shift is $\delta a_{J}=-6$ (Oe) for the external field of 200 (Oe), see Fig. 3a. When the external field is in the range between $H_{K}$ and $4 \pi M_{s}$, the precessional solutions begin to appear. Further increasing the (negative) spin torque results a new stable state, see the plateau labeled B in Fig. 3b. This new stable "B" state exists even for a single domain case as pointed out in Ref. 20]. If one applies an external field larger than $4 \pi M_{s}$, the precessional states disappear for the M-I loops. Instead, there are multiple minor loops at large current density, see Fig. 3c. 


\section{Energy dissipation and energy pumping}

The new stable states, labeled as "B" in Fig. 3b, deserve further investigation. This is because the existence of the " $\mathrm{B}$ " state is yet another signature of the effect due to spin torque; without the spin torque, the magnetization of the final stable state would be in the plane for any in-plane magnetic field and the torque $\boldsymbol{\Gamma}=\mathbf{M} \times \mathbf{H}_{\text {eff }}$ equals to zero. In Fig. 4, we show the magnetization vector pattern for the " $\mathrm{B}$ " state: there is a significant out-of-plane component. If we calculate the effective magnetic field $\mathbf{H}_{\text {eff }}$ for the "B" states, we find that the effective field is not parallel to the magnetization direction, there are angles between the two vectors $\mathbf{H}_{\text {eff }}$ and $\mathbf{M}$ for those "B" states. This would be unacceptable for the LLG without the spin torque, because the non-parallel configuration between $\mathbf{H}_{e f f}$ and $\mathbf{M}$ can not be in the static stable condition. However, in the presence of spin torque it is possible, because the static stable condition is zero total torque, $\boldsymbol{\Gamma}_{t o l}=\mathbf{M} \times \mathbf{H}_{e f f}-\frac{a_{J}}{M_{s}} \mathbf{M} \times\left(\mathbf{M} \times \hat{\mathbf{M}}_{p}\right)=$ 0 .

Another interesting observation is the variation of the magnetic energy of the system. Without the spin torque, the change of the magnetic energy of system can be easily derived from LLG equation,

$$
\frac{d E}{d t}=-\frac{\alpha \gamma}{1+\alpha^{2}} \frac{1}{M_{s}}\left|\mathbf{H}_{e f f} \times \mathbf{M}\right|^{2} .
$$

Thus, the non-parallel configuration between $\mathbf{H}_{\text {eff }}$ and $\mathbf{M}$ would continuously dissipate the energy, i.e. $\frac{d E}{d t}<0$, until the magnetic energy of system reaches the local minimum, i.e., $\mathbf{H}_{e f f} / / \mathbf{M}$. When the spin torque turns on, it is possible to compensate the loss of the energy by the gain from the spin torque,

$$
\frac{d E}{d t}=-\frac{\gamma}{1+\alpha^{2}} \frac{1}{M_{s}}\left[\alpha\left|\mathbf{H}_{e f f} \times \mathbf{M}\right|^{2}-a_{J}\left(\alpha M_{s} \hat{\mathbf{M}}_{p}-\mathbf{M} \times \hat{\mathbf{M}}_{p}\right) \cdot\left(\mathbf{H}_{e f f} \times \mathbf{M}\right)\right]
$$

where the first term is the energy loss due to damping and the second term is the energy

input (output) due to spin torque. If the first term is less than the second, i.e. $\frac{d E}{d t}>0$, the magnetic energy increases. If the first term is exactly balanced with the second, the net energy loss will be null and it is therefore possible to form a stable state "B" where $\left|\mathbf{M} \times \mathbf{H}_{e f f}\right|$ is nonzero but $\frac{d \mathbf{M}}{d t}=0$. If $\frac{d E}{d t}$ becomes a periodic function, a stable precessional state appears. 


\section{Precessional states}

As we have shown in the preceding sections, the solution of the LLG contains stable precessional states in which the magnetization never converges to a final state as if the system had no damping at all. These precessional solutions are one of the unique properties of the magnetization dynamics driven by a spin-polarized current. Without the current, the system is always losing its energy due to Gilbert damping and thus the precession can not be sustained after certain time scales, typically in a few nanoseconds. In Fig. 5, we show two trajectories of magnetization vectors at the stable precessional states for two magnetic fields but the same current. These two trajectories clearly show a large out-of-plane component of magnetization, indicating the significant role played by the demagnetization factor.

A further inspection of the magnetic energy of the precessional states reveals interesting temporal variation of the energy. The system constantly gains energy via the transfer of the angular momentum of the conduction electrons (spin currents) to the local magnetization. Thus the precessional states are the result of competition between the Gilbert energy dissipation and the spin torque energy input. The damping and pumping rates are not the same at a given time. The magnetic energy oscillates even for a steady state current. In Fig. 6, we show the evolution of magnetic energy as a function of time for different damping constants. The initial state of the system is a "leaf state" without the external field and the spin torque. At $\mathrm{t}=0$, we simultaneously apply an external field $H_{\text {ext }}=-2000(\mathrm{Oe})$ and $a_{J}=-400$ (Oe).

For a large damping constant, i.e., $\alpha>\frac{\left|a_{J}\right|}{2 \pi M_{s}}$, the energy dissipation is dominant. Thus, the system loses magnetic energy and eventually sets into a local minimum energy as shown in Fig. 6a. With decreasing $\alpha$, for example $\alpha=0.03$, the energy dissipation by damping and energy pumping by the spin torque becomes comparable. In response to the applied field and the spin torque, the magnetic energy begins to oscillate in a precessional cycle. If the damping constant further decreases, the energy oscillates around a value that increases and eventually reaches an asymptotic value. Such a dynamic change of the magnetic energy can be explained again from Eq. (10). When $\mathbf{M}_{p}$ is parallel to $\mathbf{H}_{\text {eff }}$, the rate of the energy change reduces to pure dumping or negative damping, i.e.,

$$
\frac{d E}{d t}=-\frac{\gamma}{1+\alpha^{2}} \frac{1}{M_{s}}\left(\alpha-\frac{a_{J}}{\left|H_{e f f}\right|}\right)\left|\mathbf{H}_{e f f} \times \mathbf{M}\right|^{2} .
$$

If $\alpha-\frac{a_{J}}{\left|H_{e f f}\right|}>0$ the magnetization seeks for energy minimum, otherwise, it seeks for the energy maximum, i.e., negative damping. In general, however, $\mathbf{M}_{p}$ is not parallel to $\mathbf{H}_{e f f}$ 
and thus, Eq. (10) can be positive and negative at different times and one arrives at the precessional motion.

It is interesting to observe that the frequency of the stable precession highly depends on the damping parameter; this is in sharp contrast with the normal precession where the frequency is determined by the effective field. The reason is rather simple: the stable precessional states described here are from the competition between the damping and spin torque, and they are not the initial precessional motion (which is unstable) driven by the effective field. In Fig. 7, we show the precessional frequencies as a function of damping constant $\alpha$. As expected, the frequency monotonically increases as one decreases the damping parameter. For the same reason, the frequency increases with increasing spin torques.

To further analyze these precessional states, we have shown, in Fig. 8 the temporal evolution of three magnetization components when the magnetization reaches a stable precessional state. The magnetization shows significant out-of-plane component (z-direction) during the oscillation. The swing of magnetization in the in-plane hard axis direction is as large as that in the easy axis direction. A noticeable difference between low temperature and room temperature is the difference for the out-of-plane component of magnetization. There are two stable precessional solutions at the given external field and spin torque. One is the precession at the upper half plane, i.e., $M_{z}>0$, as shown in Fig. 8a. Another solution is located at the lower half plane, i.e., we simply replace $M_{z}$ by $-M_{z}$ and keep the $x$ and $y$ components as in Fig. 8a. These are degenerated solutions and the magnetization dynamics takes either one of the two solutions but not both at zero temperature. At room temperature, however, these two solutions can jump around, making the out-of-plane component crossing the $\mathrm{x}-\mathrm{y}$ plane. This thermally activated transition between two precessional states originates from low barrier heights-the thermal energy at room temperature can not be ignored in this small structure, as shown in Fig. 8b. While the thermal energy can not wash out the precessional solutions, it does alter the magnetization dynamics significantly.

\section{E. Switching speed}

We have shown that there is a critical spin torque to switch the magnetization. We now address how fast the switching is. The switching speed has been analytically calculated for a single domain sphere and a single domain thin film, in the absence of the spin torque, 
i.e., switching by the external field. The speed depends on the damping parameter. The optimal damping parameter for the fastest switching is $\alpha=1$ for the sphere and $\alpha \approx 0.013$ for the thin film [29]. Our question is what is the optimal damping parameter for the spin torque induced switching? How fast the switching speed compared with the field induced switching?

Let us first take a look at the influence of the spin torque on the switching speed by a magnetic field. The initial magnetization ("leaf state") is at the positive $\mathrm{x}$-direction. A reversed magnetic field is applied in the negative $\mathrm{x}$-direction at $t=0$. When the $\mathrm{x}$-component of the magnetization $\left\langle M_{x}>\right.$ reaches at least -0.95 and it stays below this value after $t_{s}$, we define this $t_{s}$ as the switching time. In Fig. 9, we show the dependence of the switching time for three different spin torques. The switching time is enhanced for one direction of the current and is shortened for the opposite direction. We have noticed that the optimal damping parameter varies when the spin torque changes.

To study the switching speed due to spin torque, we must apply a spin torque exceeding the critical spin torque. In Fig. 10, we show the switching time after a spin torque larger than the critical spin torque is applied. The switching time is very slow as the spin torque approaches the critical spin torque. We find that the switching time can be reasonably fitted by $t_{s}^{-1} \propto=a_{J}-a_{c r i t}$. Since the critical spin torque $a_{c r i t}$ is proportional to the damping parameter, the applied spin torque is shifted for different damping parameters as shown in Fig. 10.

Up till now, we have concentrated our description of the spin torque in the limit of the steady state current. It is, however, desirable to see the magnetization dynamics in the case of a pulsed form. Here we should consider a simple form of the spin torque pulse: a square pulse whose width is $t_{w}$, i.e., we will ignore the rise and fall times of the pulse; in real devices, one should also consider these times. For such a pulsed electrical current, one would expect that whether the magnetization can be switched by the spin torque depends on pulse's amplitude and width. For a fixed pulse amplitude larger than the critical spin torque, there is minimum pulse width needed to achieve current-driven magnetization reversal. In Fig. 11, we show the magnetization dynamic after the pulse of $a_{J}=2500(\mathrm{Oe})$ is applied at $t=0$. In the first two panels, the durations of the pulse are just not enough to fully reverse the magnetization, i.e., the magnetization returns to the initial state $\left(<M_{x}>\approx 1.0\right)$ after one removes the current. Just a small increase of the pulse width, see Fig. 11c, the magnetization 
is able to fully reverse itself after the signal is taken away. Thus, $t_{w}=0.775$ (ns) is defined as the minimum width required for the current-induced magnetization reversal for the pulse amplitude $a_{J}=2500$ (Oe).

The minimum pulse width defined above depends on the pulse amplitude. With increasing pulse amplitude, the minimum pulse width decreases. We can construct a phase diagram of magnetization reversal by varying the pulse amplitude and repeating the procedure to determine the minimum pulse width in each case. As shown in Fig. 12, the minimum pulse width increases dramatically for the pulse amplitude close to the critical spin torque; this phenomenon is very similar to the classical "critical slowing down" in statistical physics: when an external force approaches a critical value, the system slows down. In our case, we find that the reversal time is mainly spent at the beginning stage of switching.

The minimum pulse width is also affected by other parameters. In Fig. 13, the computed reversal/non-reversal phase diagram is plotted in terms of the damping constant for several external magnetic fields. Since the thin film anisotropy field is about 500 Oe, the largest external fields $\left(\left|H_{\text {ext }}\right| \leq 200 \mathrm{Oe}\right)$ used in Fig. 13 can not trigger the magnetization reversal itself. The increase of the minimum width for a larger damping constant is consistent with the picture that the spin torque is competing with the damping process. One interesting feature in Fig 13 is that the external field affects the minimum width much more significantly for large damping parameters than for small ones. This is due to the fact that the spin torque $\left(a_{J}=2500 \mathrm{Oe}\right)$ is closer to the critical spin torque for larger damping constant. By applying the magnetic field, one can shift the critical spin torque to smaller values. Therefore, the minimum width is much smaller compared to that without the magnetic field.

\section{F. Thermal switching}

Finally, we address the problem of thermally activated magnetization switching in the presence of the spin torque. As the device approaches nanometer size, temperature driven magnetization reversal becomes one of key factors limiting the device performance [30, 31, 32]. We have already seen in Fig. 8b that the inclusion of the thermal activation has significantly altered the magnetization dynamics.

The thermally assisted magnetization reversal is conventionally modeled by the Néel-

Brown formula $\tau=f_{0}^{-1} \exp \left(E_{b} / k_{B} T\right)$ where $\tau$ is the thermal switching time, $E_{b}$ is the 
energy barrier, and $f_{0}$ is the attempt frequency of the order of $10^{9}\left(\mathrm{~s}^{-1}\right)$. For a single domain particle, the energy barrier is simply $E_{b}=E_{0}\left(1-H / H_{c}\right)^{\beta}$ where $H_{c}$ is the coercive field and $E_{0}$ is an extrapolation of the energy barrier at zero field. Notice that the energy barrier is independent of the damping parameter. If the spin torque is purely competing with the damping, one would expect that the spin torque does not affect the energy barrier and thus the component $\beta$ would be independent of the spin torque. We have found, however,

the spin torque does more than altering the damping parameter as shown below. Let us suppose the magnetization of the free layer is initially saturated in the positive $x$ direction. At $t=0$, we apply a negative magnetic field which is close to but less than the switching field $H_{c}$. At the same time, a spin torque is also applied to the system. At the finite temperature, the average waiting time for the magnetization reversal is given by the Arrhenius law, the switching probability decays as $e^{-t / \tau}$. To minimize the statistic error, we determine the relaxation time (average waiting time) by repeating the above procedure 800 times for each spin torque. In Fig. 14, we show the probability of the free layer not being switched $\left(1-P_{s}^{e x p}(t)\right)$ as a function of the relaxation time $t$ for a magnetic field $H_{\text {ext }}=-438$ (Oe). The reversal probability $P_{s}^{e x p}(t)$ increases with spin torque; the positive spin torque $\left(a_{J}>0\right)$ leads to a faster thermal switching and the negative spin torque results in slower thermal reversal. The distribution of the relaxation time can be well-fitted to the Arrhenius formula function, but now the energy barrier depends on the spin torque: the positive current favors lower energy barrier. We show the fitted relaxation time as a function of the spin torque for two different external fields in Fig. 15. The linear relation indicates the effective energy barrier is linearly dependent on the current. Such results can be potentially very useful: one can control the energy barrier of a nanoscale magnetic element by applying a proper spin polarized current so that superparamagnetic effects can be overcome.

\section{DISCUSSIONS AND CONCLUSIONS}

The spin torque in LLG equation adds a new degree of freedom to control and manipulate magnetization dynamics. The spin torque differs from the other common torques: the one due to the effective field and the another one due to the dissipation (Gilbert damping). The torque from the effective field is a conservative torque in the sense that the magnetic energy is conserved while the dissipative torque produces energy dissipation as long as the 
magnetization is not static. The spin torque can play the role of the effective field as well as the dissipation torques; it is this peculiar feature making the dynamics and hysteresis loops quite unusual. We now summarize our results below.

First, in our thin sample with the dimension of $64 \mathrm{~nm}$ by $64 \mathrm{~m}$ by $2.5 \mathrm{~nm}$, the hysteresis loops and the dynamics behave qualitatively as a single domain. For example, the magnetization of each grid never differs by more than $5^{\circ}$ during any dynamic processes of the magnetization reversal (except at very high magnetic fields, $H_{\text {ext }}>4 \pi M_{s}$ when the spin wave excitations are generated; we will address the effect of extremely high field elsewhere). Therefore, most of the conclusions obtained from the analytical work where the layer is treated as a single domain are justified in the present study. However, the non-single domain feature is important when one addresses the questions such as the reversal speed. For example, in the single domain picture, one would never switch the magnetization by a large magnetic field or a large spin torque if the field and the magnetization directions are exactly at the easy axis, because there will be no initial torque in this case. If one takes the full micromagnetics into account, the magnetization at the center of the sample differs slightly from that at the edges with some characteristic frequencies. Such slight non-uniformity of the magnetization is just enough to allow spin-torques to reverse magnetization. Therefore, one should be cautious in utilizing the single domain picture in addressing the detailed dynamics of magnetization reversals.

The second conclusion we wish to emphasize is the unique feature of the spin torque: the new equilibrium states and the stable precessional states. These states would not be stable without the spin torque since the energy would be damped as long as the magnetization vector is not parallel to the direction of the effective magnetic field. This is an analogy to the classical moving object: the object will eventually come to rest if no power is added to the object because air resistance would consume all the kinetic energy of the object. Here, the spin torque is an external power supplied to the system. With appropriate conditions, i.e, with proper magnetic fields and damping constants, the stable precessional states appear, which is again analogous to the "resonant" states of classical mechanics. We have found that these stable precessional states are indeed very stable: they exist even if there is large thermal fluctuation and they appear to exist for a wide range of parameter space.

Next we have studies the influence of the spin torque on switching speed. We have found that the spin torque can enhance and retard the switching speed. The enhancement is large, 
usually a few times faster. If one wishes to have an order of magnitude increase, one should consider the spin torque and magnetic field applied in other directions, not the direction of the easy axis. It has been known that the hard axis application of these components can dramatically increase the switching speed. One can easily explore the best scenario for the ultra fast switching devices by changing directions of the spin torque. Finally, we have pointed out that the effective energy barrier for thermally assisted reversal can be influenced by the spin torque. This result further illustrates the fundamental difference between the spin torque and the damping.

In conclusion, we have shown the various dynamic behaviors in the presence of the spin torque. We hope that this work can generate more experimental efforts to verify those novel dynamic phenomena unique to the spin torque. This work is supported by NSF (ECS0223568) and INSIC.

[1] L. Berger, Phys. Rev. B 54, 9359 (1996); 59, 11465 (1999).

[2] J. Slonczewski, J. Magn. Magn. Mater. 159, L1 (1996); 195, L261(1999).

[3] Ya B. Bazaliy, B. A. Jones, and S. C. Zhang, Phys. Rev. B 57, R3213 (1998).

[4] X. Waintal, E. B. Myers, and P. W. Brouwer, Phys. Rev. B 62, 12317 (2000).

[5] M. D. Stiles and A. Zangwill, Phys. Rev. B66, 014407 (2002); J. Appl. Phys. 91, 6812 (2002).

[6] A. Brataas, Y. V. Nazarov, and G. E. Bauer, Phys. Rev. Lett. 84, 2481 (2000).

[7] S. Zhang, P. M. Levy, and A. Fert, Phys. Rev. Lett. 88, 236601 (2002).

[8] J. Slonczewski, J. Magn. Magn. Mater. 247, 324 (2002).

[9] M. Tsoi, A. G. M. Jansen, J. Bass, W.-C. Chiang, M. Seck, V. Tsoi, and P. Wyder, Phys. Rev. Lett. 80, 4281(1998).

[10] E. B. Myers, D. C.Ralph, J. A. Katine, R. N. Louie, and R. A. Buhrman, Science 285, 867 (1999).

[11] J. Z. Sun, J. Mag. Mag. Mater. 202, 157 (1999).

[12] J. A. Katine, F. J. Albert, R. A. Buhrman, E. B. Myers, and D. C. Ralph, Phys. Rev. Lett. 84, 3149 (2000).

[13] J. Grollier, V. Cros, A. Hamzic, J. M. George, H. Jaffres, A. Fert, G. Faini, J. Ben Youssef, and H. Legall, Appl. Phys. Lett. 78, 3663 (2001). 
[14] F. J. Albert, J. A. Katine, R. A. Buhrman, and D. C. Ralph, Appl. Phys. Lett. 81, 2202 (2002).

[15] J. E. Wegrowe, D. Kelly, Y. Jaccard, Ph. Guittienne, and J. Ph. Ansermet, Europhys. Lett. 45, 626 (1999); J. E. Wegrowe, X. Hoffer, Ph. Guittienne, A. Fabian, L. Gravier, T. Wade, and J. Ph. Ansermet, J. Appl. Phys. 91, 6806 (2002).

[16] M. Tsoi, A. G. M. Jansen, J. Bass, W.-C. Chiang, V. Tsoi, P. Wyder, Nature (London) 406, 46 (2000).

[17] J. Z. Sun, D. J. Monsma, M. J. Rooks, and R. H. Koch, Appl. Phys. Lett. 81, 2202 (2002).

[18] E. B. Myers, F J. Albert, J.C. Sankey, E. Bonet, R. A. Buhrman and D. C.Ralph, Phys. Rev. Lett. 89196801 (2002).

[19] J. Z. Sun, Phys. Rev. B 62, 570 (2000).

[20] Ya. B. Bazaliy, B. A. Jones, and S. Zhang, cond-mat/000934 (unpublished); J. Appl. Phys. 89, 6793 (2001).

[21] J. Miltat, G. Albuquerque, A. Thiaville, and C. Vouille, J. Appl. Phys. 89, 6982 (2001).

[22] R. P. Cowburn and M. E. Welland, Appl. Phys. Lett. 72, 2041 (1998).

[23] C. H. Back, R. Allenspach, W. Weber, S.S.P.Parkin, D.Weller, E.L.Garwin, and H.C. Siegmann, Science 285, 864(1999).

[24] R. Urban, G. Woltersdorf, and B. Heinrich, Phys. Rev. Lett. 87, 217204 (2001).

[25] Y. Tserkovnyak, A. Brataas, and G. E. W. Bauer, Phys. Rev. Lett. 88, 117601(2002).

[26] J. L. Garcia-Palacios and F. J. Lazaro, Phys. Rev. B 58, 14937(1998).

[27] W. Scholz, T. Schrefl, and J. Fidler, J. Magn. Magn. Mater. 233, 296 (2001).

[28] U. Nowak, Ann. Rev. of Comp. Phys. 9, 105 (2001).

[29] R. Kikuchi, J. Appl. Phys. 27, 1352(1956).

[30] R. H. Koch, G. Grinstein, G. A. Keefe, Y. Lu, P. L. Trouilloud, W. J. Gallagher, and S. S. P. Parkin, Phys. Rev. Lett. 84, 5419 (2000).

[31] N. D. Rizzo, M. DeHerreta, J. Janesky, B. Engel, J. Slaughter, and S. Tehrani, Appl. Phys. Lett. 80, 2335 (2002).

[32] W. Wernsdorfer, E. B. Orozco, K. Hasselbach, A. Benoit, B. Barbara, N. Demoncy, A. Loiseau, H. Pascard, and D. Mailly, Phys. Rev. Lett. 78, 1791 (1997). 
FIG. 1: Schematic of the pillar device with a positive spin current defined as the electrons flowing from the thinner to the thicker Co layers (opposite direction for the current flow).

FIG. 2: Hysteresis loops of the free Co layer in the presence of the different spin torques: (a) $a_{J}=-100(\mathrm{Oe}) ;(\mathrm{b}) a_{J}=-200(\mathrm{Oe}) ;(\mathrm{c}) a_{J}=-400(\mathrm{Oe})$.

FIG. 4: Magnetic moments at the point "B" in Fig.3(b), where $H_{e x t}=-2000$ Oe, $a_{J}=-3000$ (Oe).

FIG. 5: The trajectories of two stable precessional states that are within the shaded area of Fig. 2c, where $a_{J}=-400$ Oe.

FIG. 6: Temporal evolution of the magnetic energy for three different damping constants $\alpha(=$ $0.1,0.03,0.008)$. The external field is $H_{e x t}=-2000$ Oe and spin torques $a_{J}=-400(\mathrm{Oe})$.

FIG. 7: Precessional frequencies $\omega$ as a function of damping constant $\alpha$ for different $H_{\text {ext }}$ and $a_{J}$ : $H_{\text {ext }}=-1000 \mathrm{Oe}, a_{J}=-500$ (Oe) (open triangle); $H_{\text {ext }}=-2000$ Oe, $a_{J}=-500$ (Oe) (open circle); $H_{e x t}=-1000$ Oe, $a_{J}=-1000$ (Oe) (solid circle).

FIG. 8: Temporal evolution of the total magnetization components for a stable precessional state. (a) $\mathrm{T}=0 \mathrm{~K}$; (b) $\mathrm{T}=300 \mathrm{~K}$. The magnetic field is taken as -2000 Oe and spin torque $a_{J}=-400$ (Oe) that is within the shaded area of Fig.2(c).

FIG. 9: Swithing speed vs damping constant $\alpha$ for three spin torques $a_{J}=+100$ (Oe) (dashed line), $a_{J}=0$ (solid line) and $a_{J}=-100$ (Oe) (dotted line). The external field is taken as -510 Oe.

FIG. 10: Switching speed vs spin torque (larger than the critical spin torque) at $H_{\text {ext }}=0.0$ (Oe) for different damping constants.

FIG. 3: M-I loops with different external fields: (a) $H_{\text {ext }}=-200$ Oe; (b) $H_{\text {ext }}=-2000$ Oe; (c) $H_{\text {ext }}=-20000$ Oe. 
FIG. 11: Time evolution of the magnetization component $M_{x}$ with three different pulsed torque widths (0.75 ns, $0.765 \mathrm{~ns}$ and $0.775 \mathrm{~ns})$. The pulsed amplitude is 2500 (Oe) and $H_{\text {ext }}=0.0$ (Oe).

FIG. 12: Minimum pulsed spin torque width versus amplitude for zero external field.

FIG. 13: Reversed/not-reversed boundaries as a function of damping parameter for different external fields: $H_{e x t}=0$ Oe (open triangle), $H_{e x t}=-100$ Oe (full square) and $H_{e x t}=-200$ Oe (open circle). The pulsed amplitude is taken as 2500 (Oe).

FIG. 14: Probability of not being switched magnetization as a function of time at $\mathrm{T}=300 \mathrm{~K}$ for different spin torques. The solid lines are fits to the function $e^{-t / \tau}$.

FIG. 15: The relaxation time $\tau$ (solid line) and the switching probability at $t=50 \mathrm{~ns}$ (dashed line) as a function spin torque at $\mathrm{T}=300 \mathrm{~K}$ for two different external fields. 


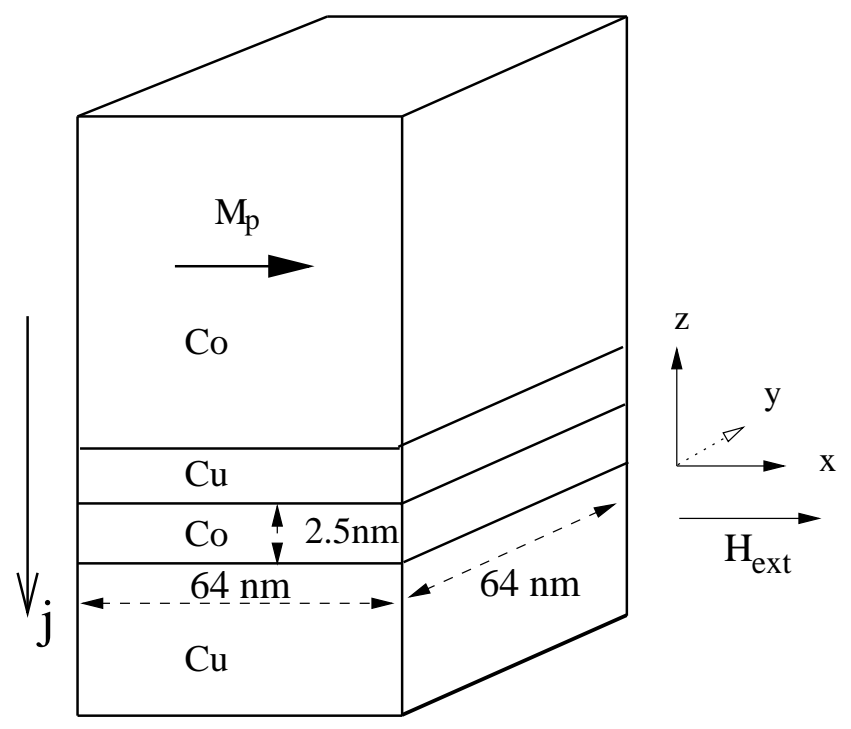

Li and Zhang Fig.1 

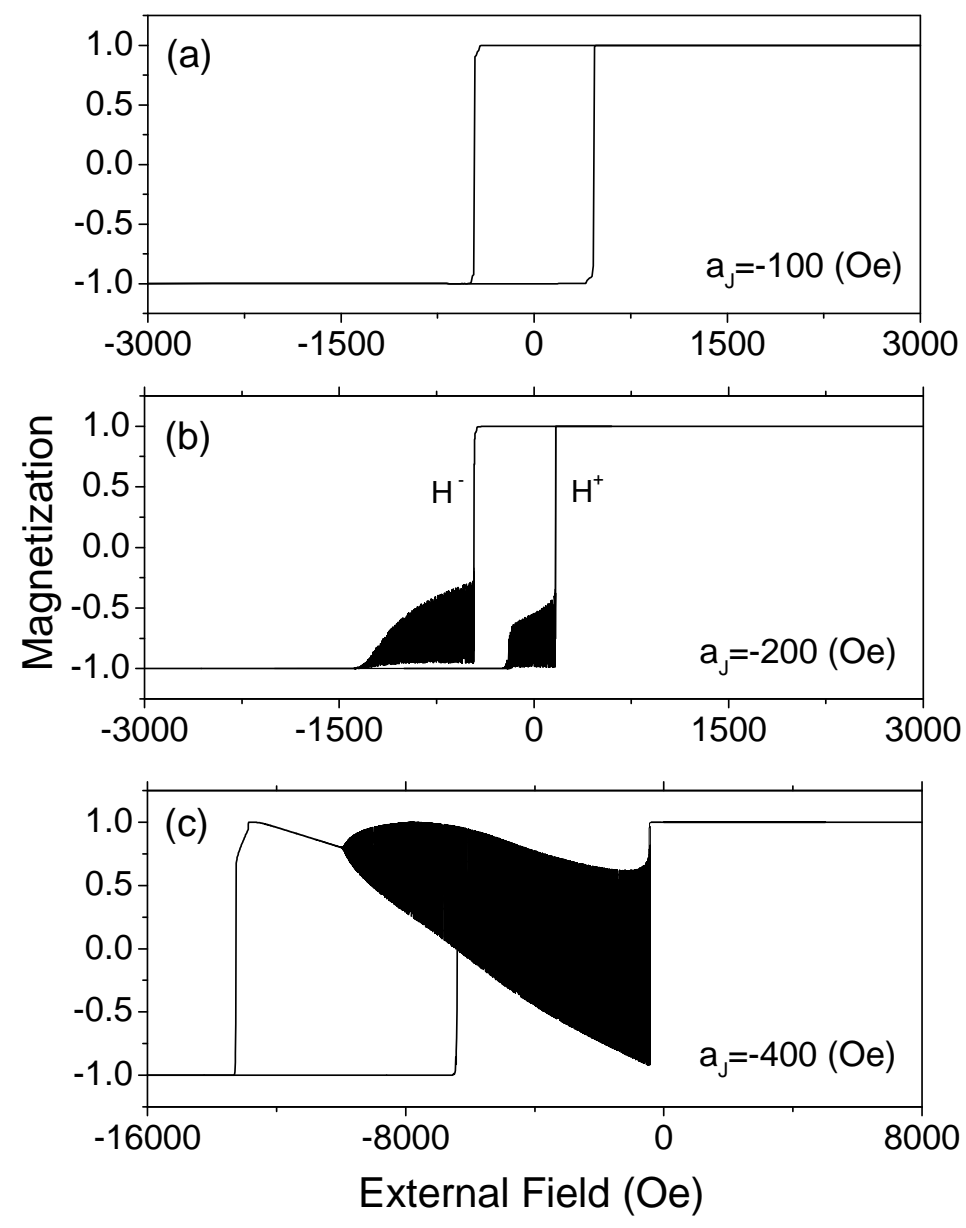

Li and Zhang Fig.2 

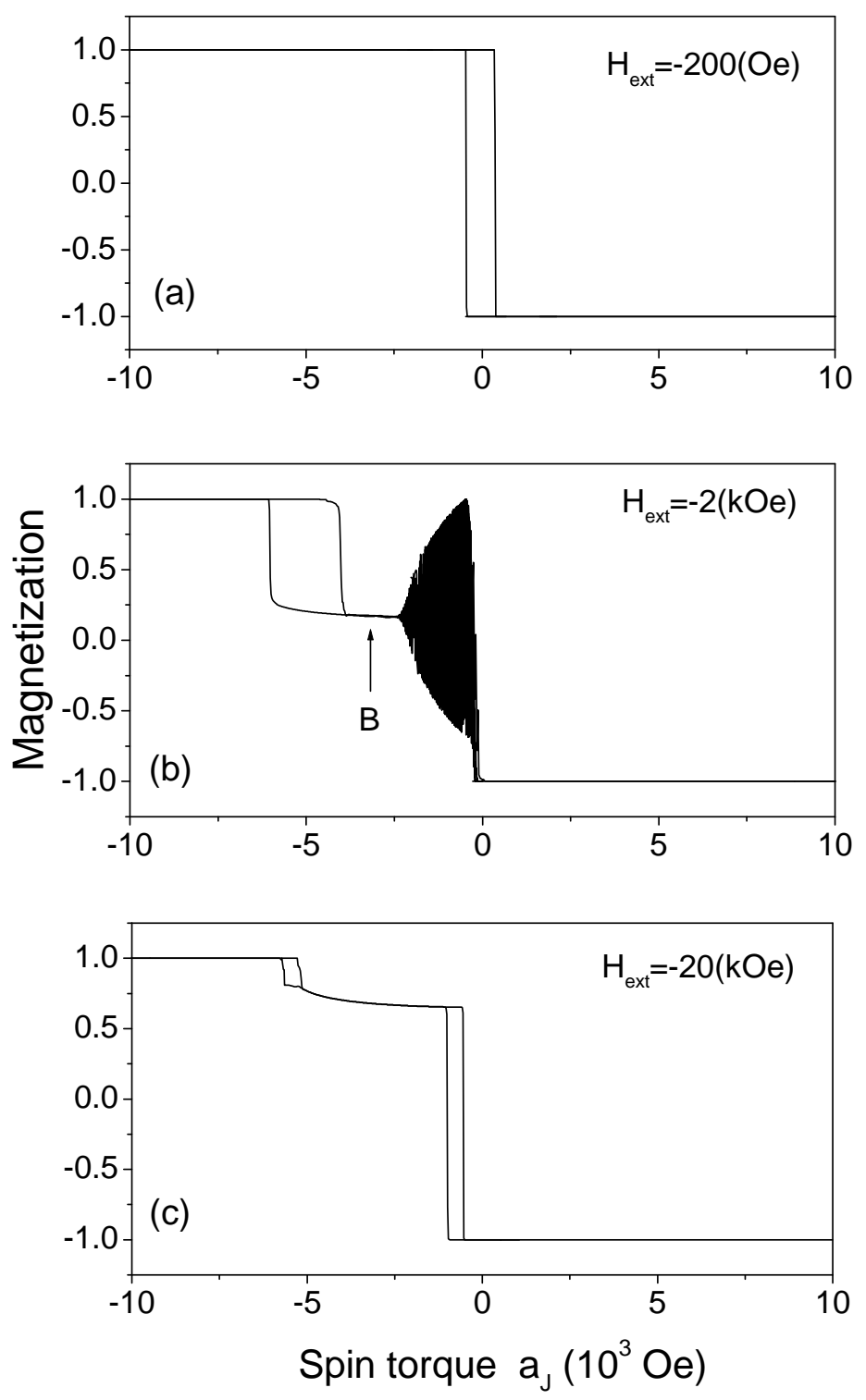

Li and Zhang Fig.3 


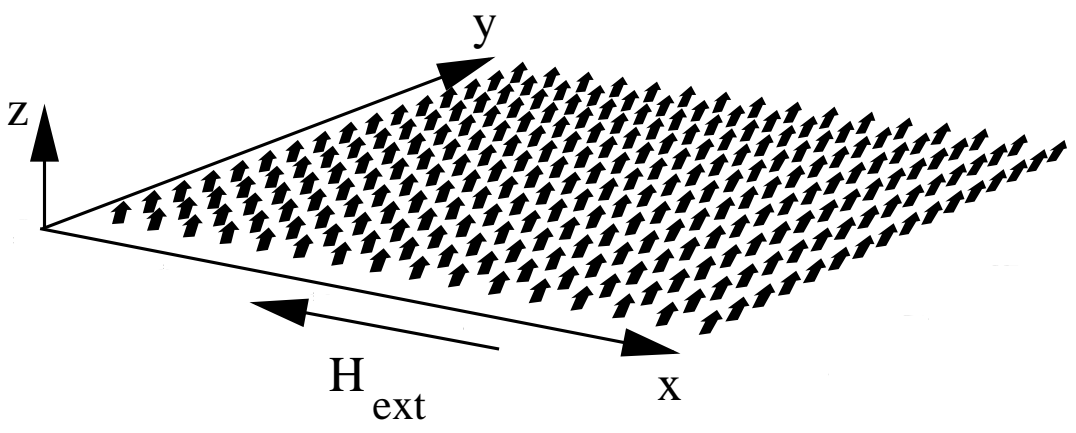

Li and Zhang Fig.4 


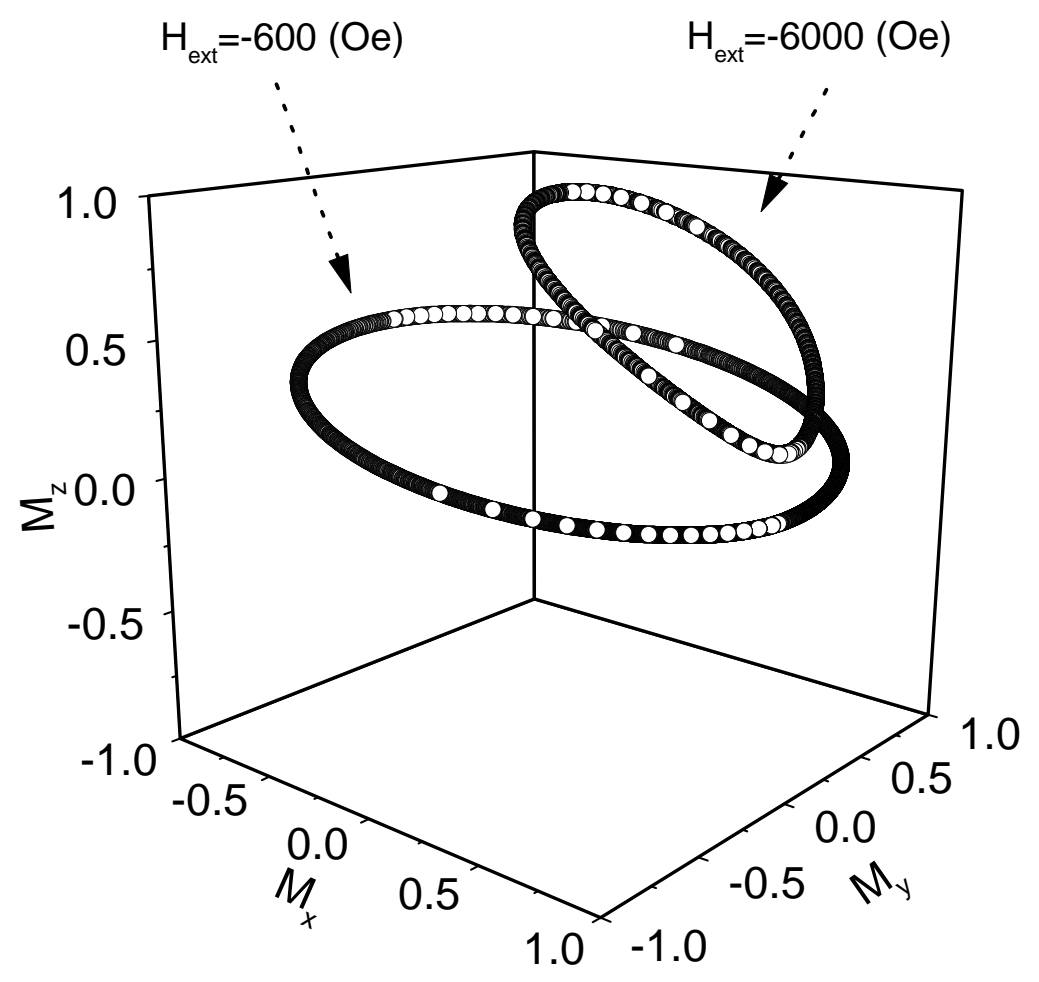

Li and Zhang Fig.5 


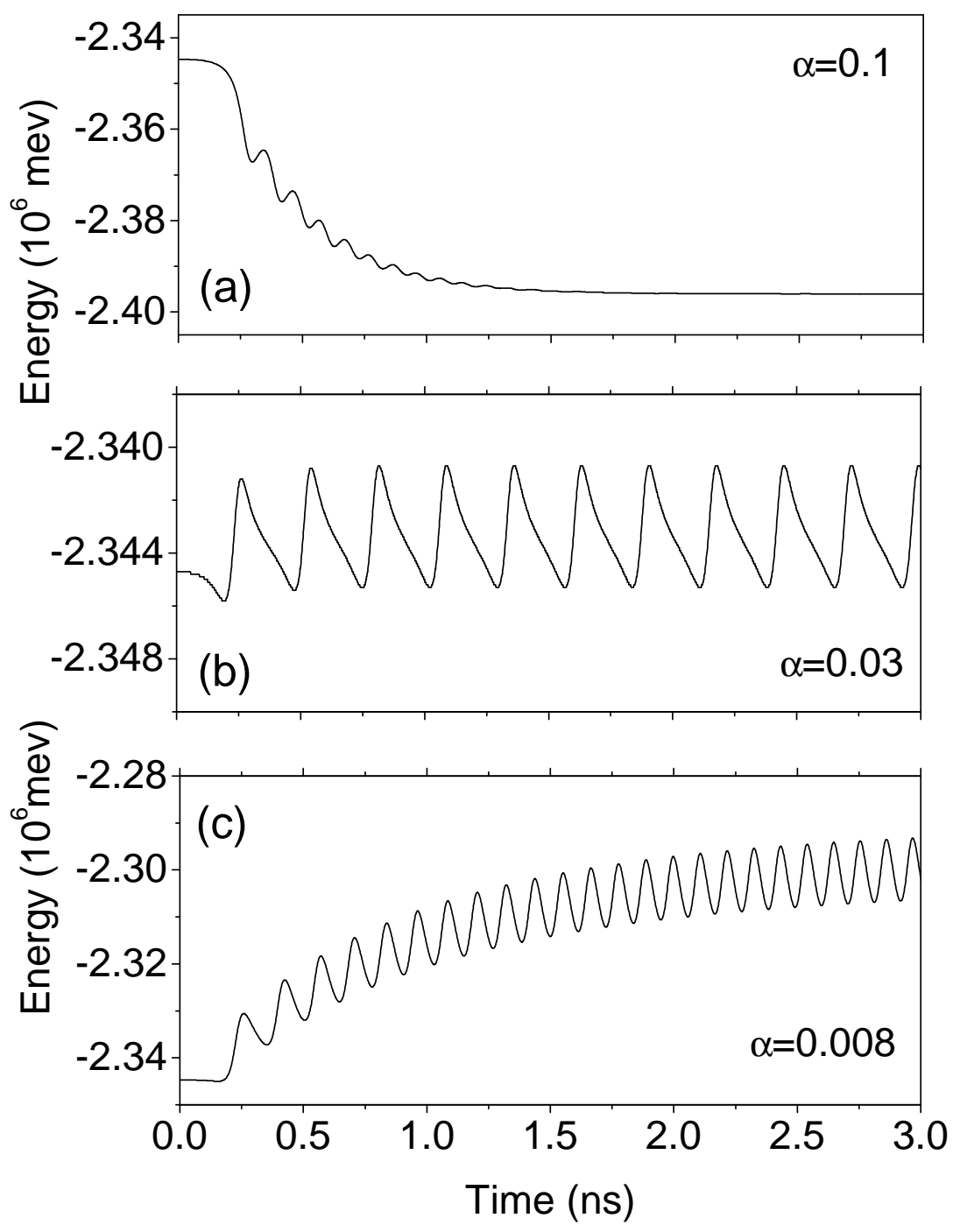

Li and Zhang Fig.6 


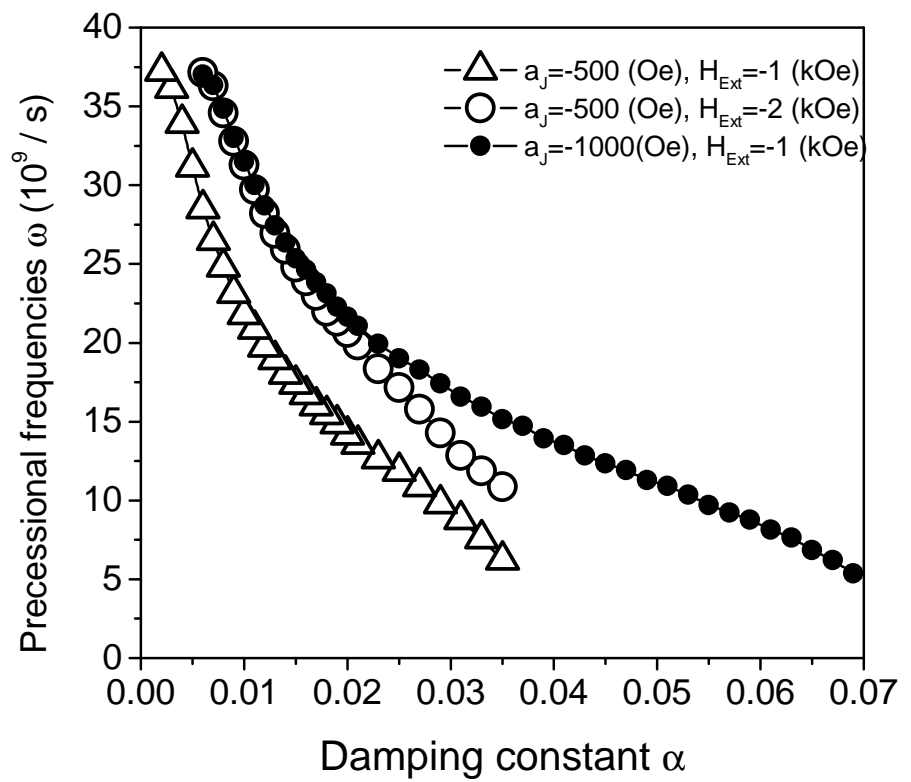

Li and Zhang Fig.7 


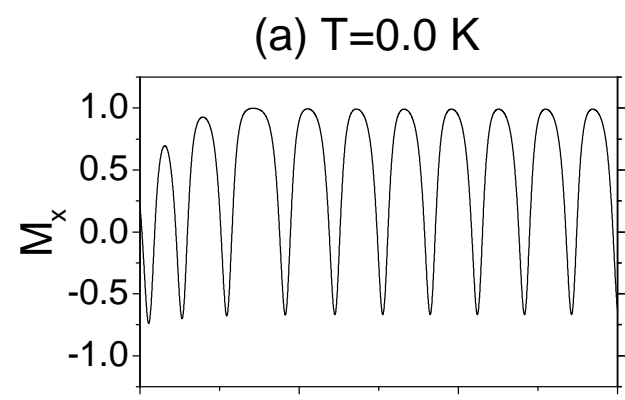

(b) $T=300.0 \mathrm{~K}$
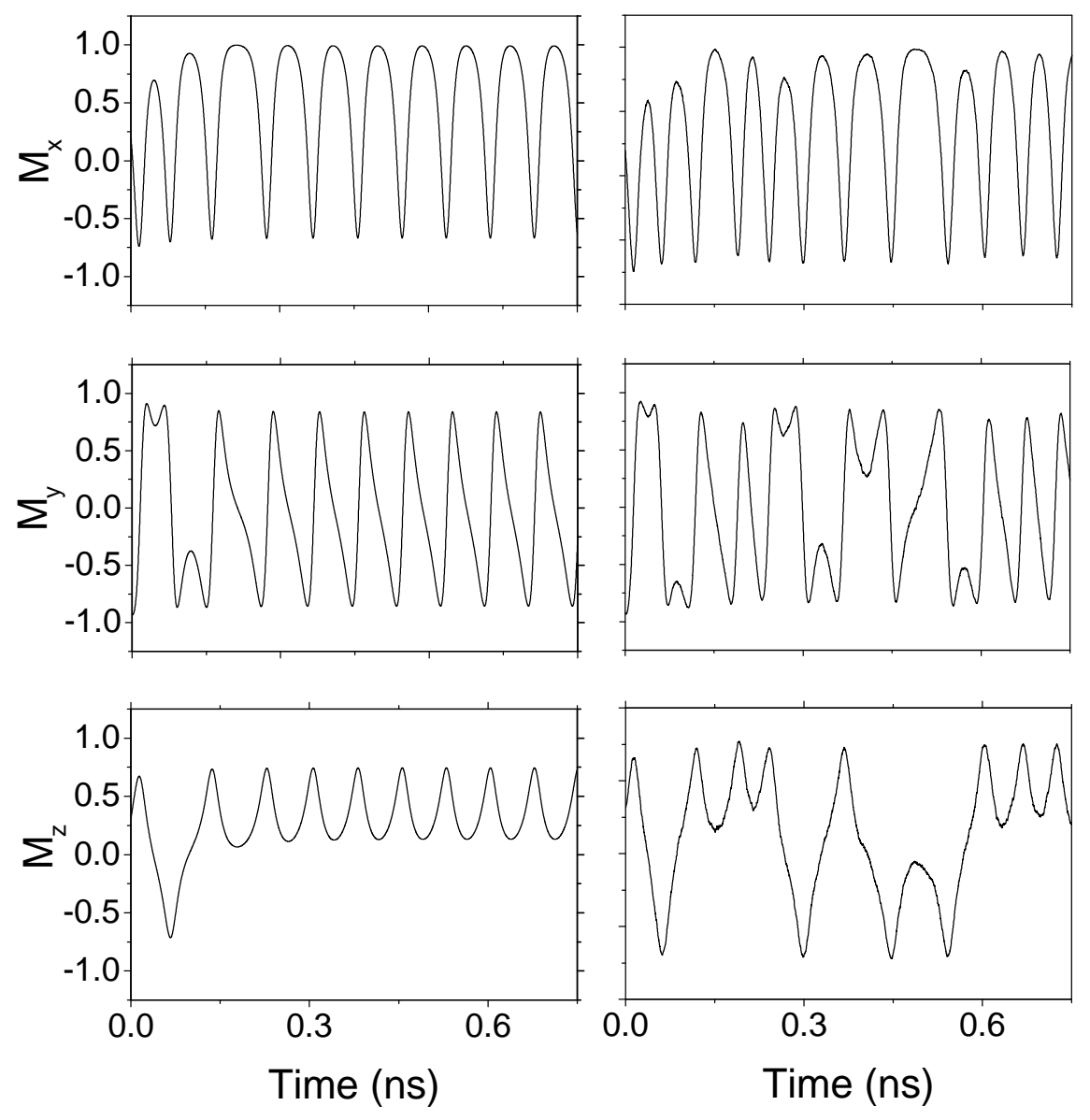

Li and Zhang Fig.8 


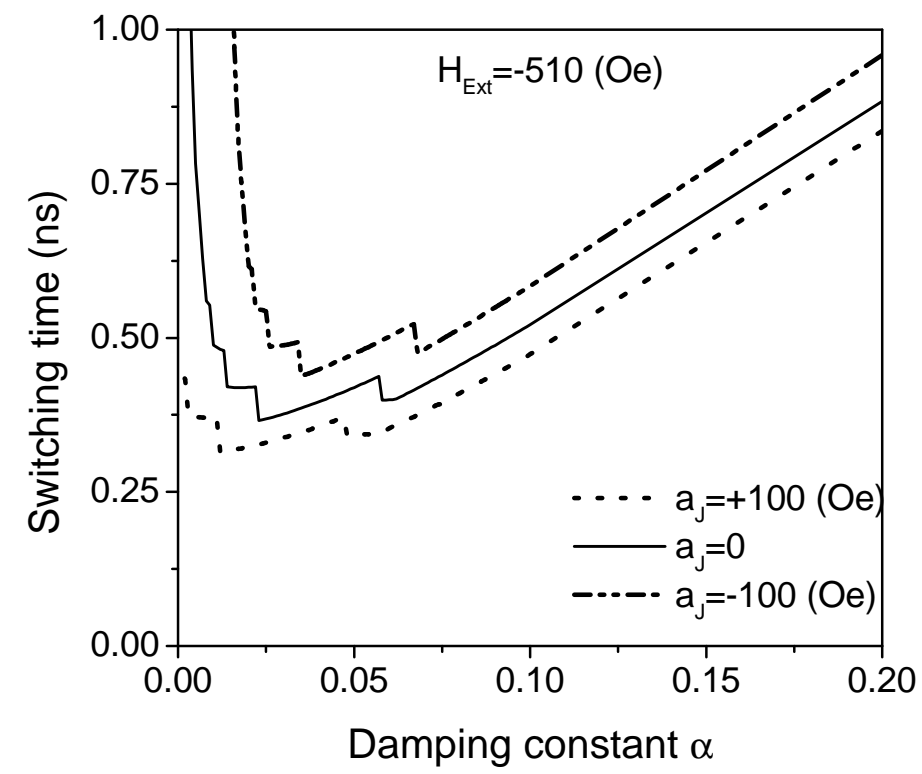

Li and Zhang Fig.9 


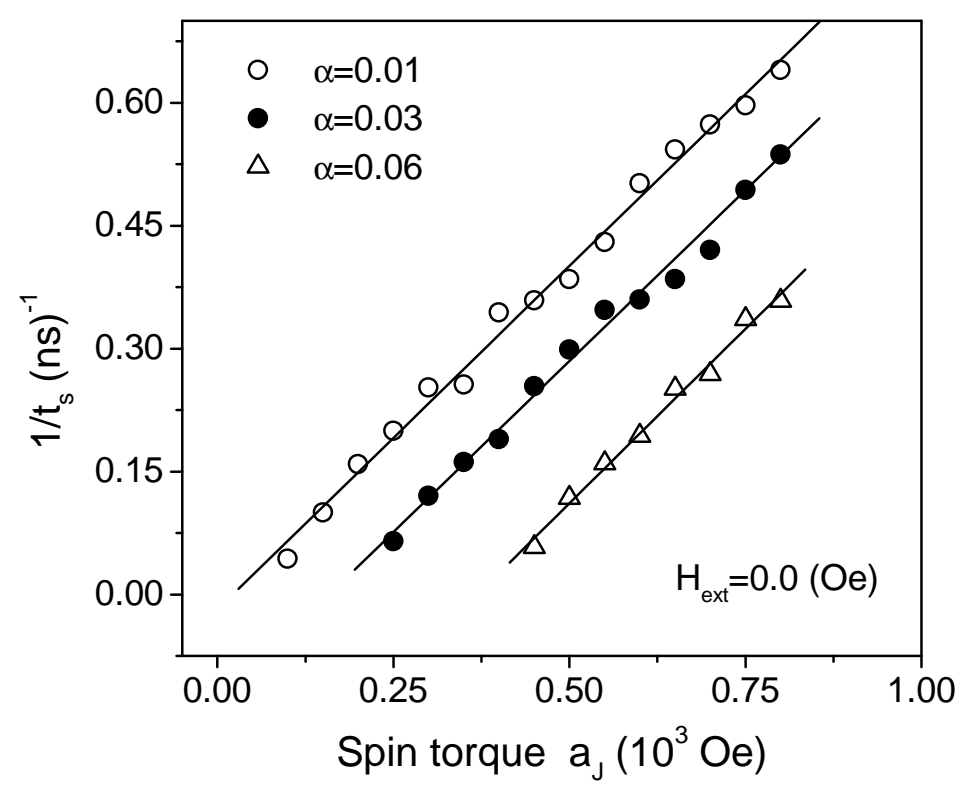

Li and Zhang Fig.10 

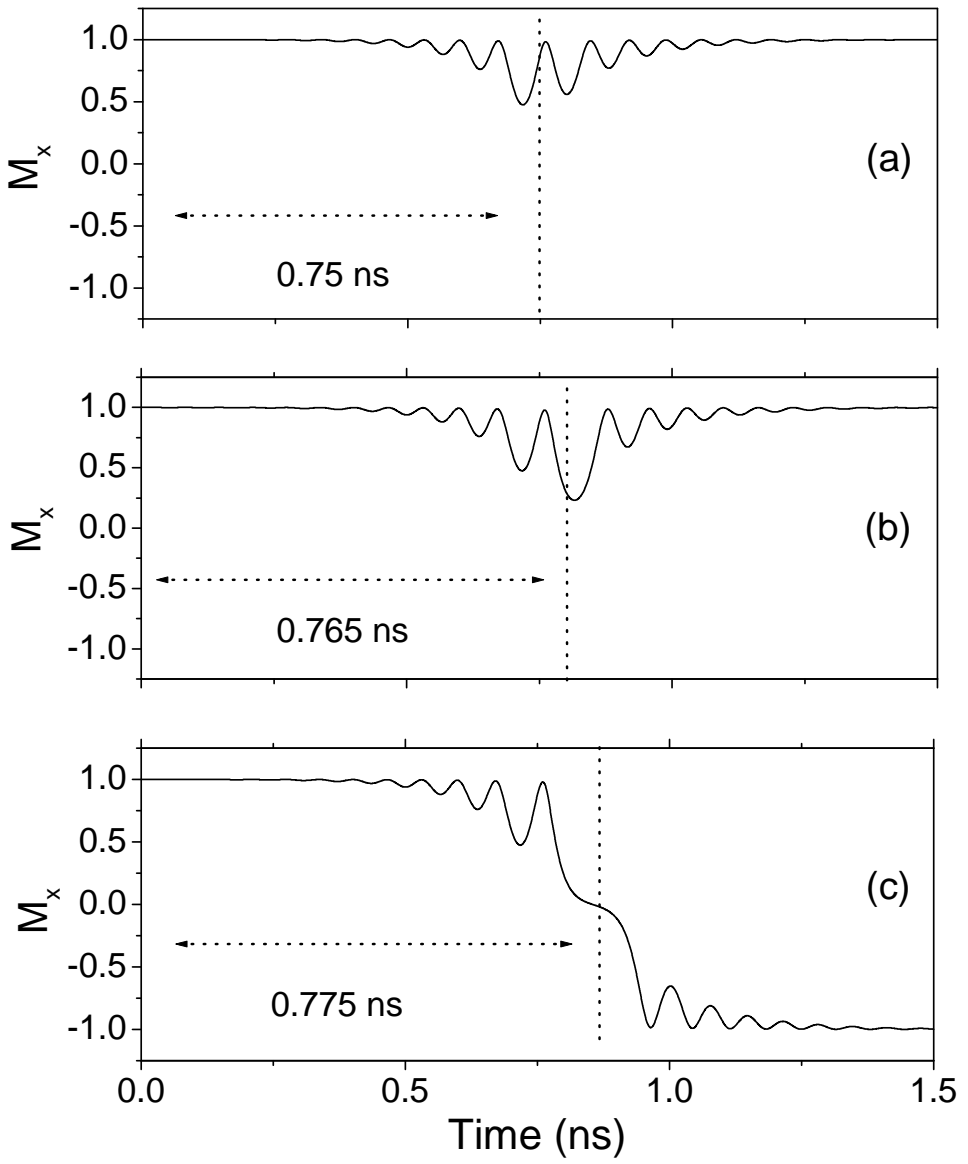

Li and Zhang Fig.11 


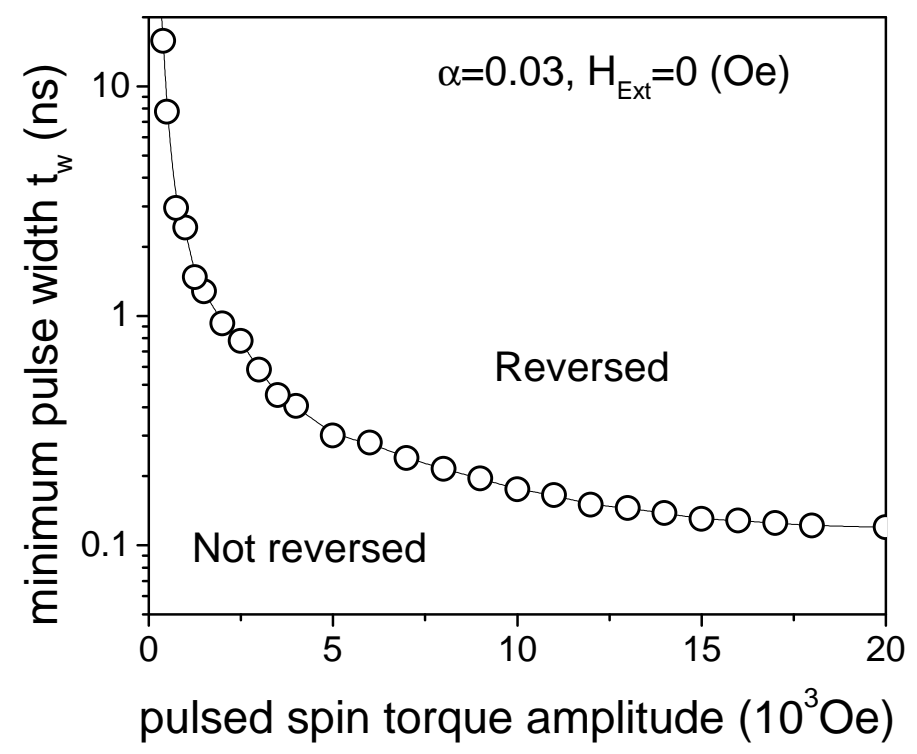

Li and Zhang Fig.12 


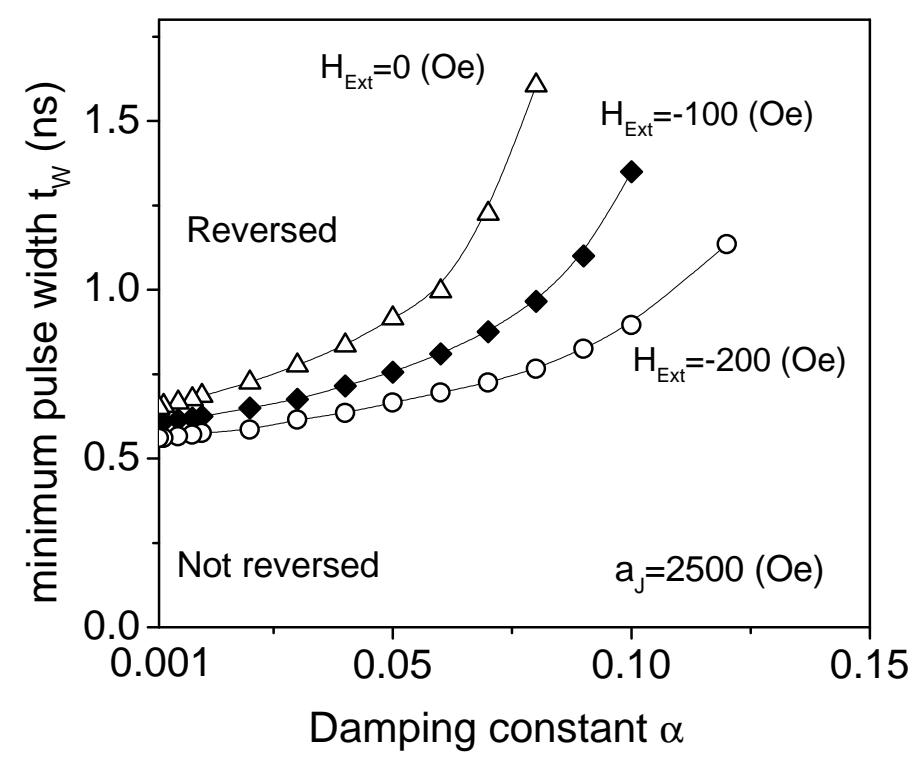

Li and Zhang Fig.13 


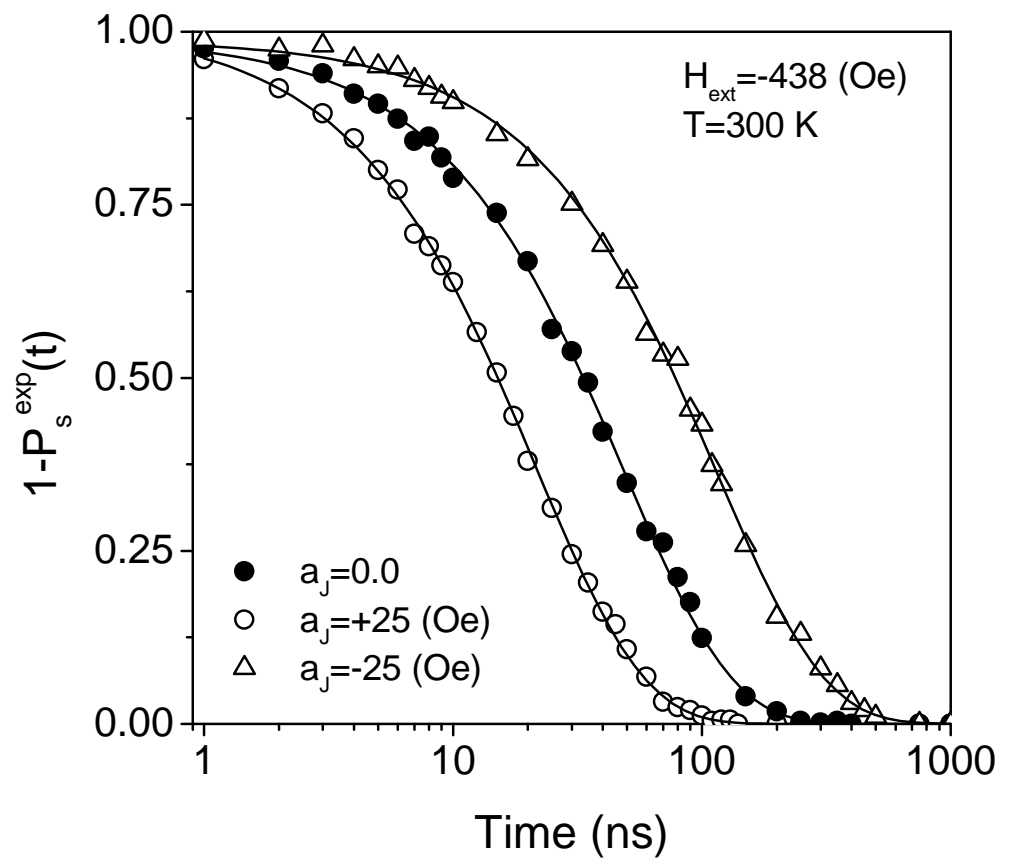

Li and Zhang Fig.14 


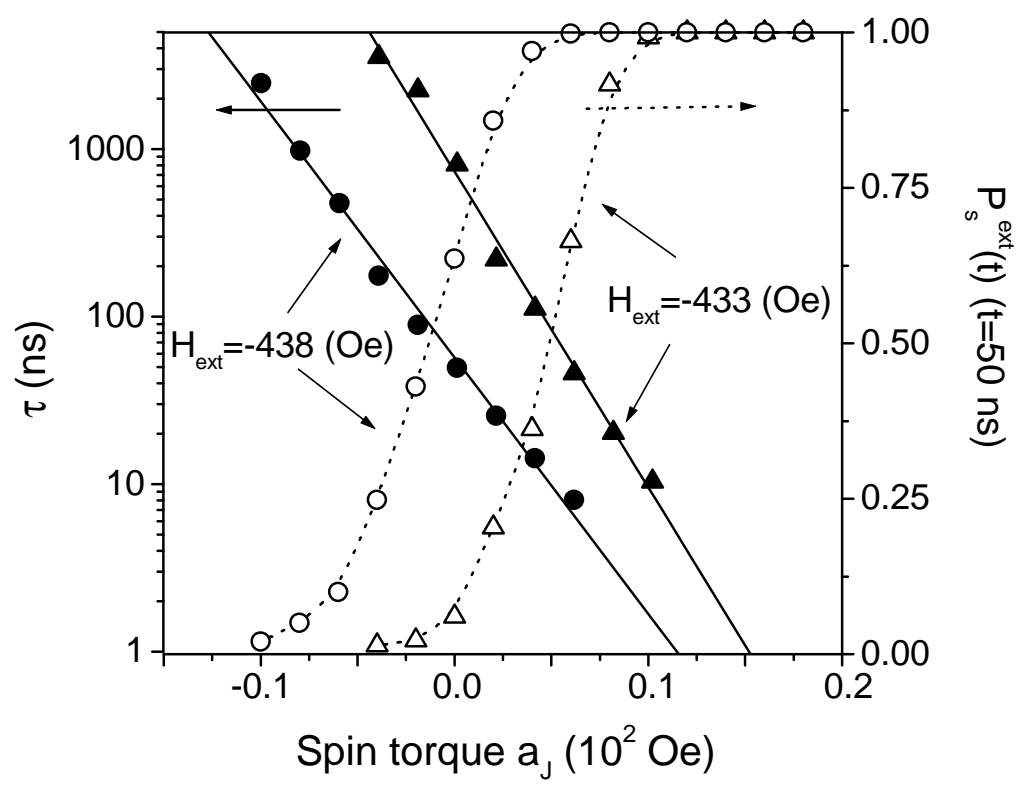

Li and Zhang Fig.15 